\title{
Long-term pacing with an inductive coupling system
}

\author{
Malcolm Clarke, David W. Evans, and B. B. Milstein \\ From the Regional Cardiac Unit, Papworth Hospital, Cambridge
}

The inductive coupling system has been used at a Regional Cardiac Unit for long-term cardiac pacing during the past 33 months. The first 44 patients so treated are described. Thirty-nine patients had endocardial electrodes and 5 had myocardial wires (2 ventricular, 3 atrial). There was I early postoperative death and 4 late deaths, 2 of which were expected. The inductive coupling system is relatively inexpensive and has the great advantage of an easily interchangeable external generator, with variable rate control and adjustable power setting. Battery changing is easily and safely effected by the patient and no special electronic testing or follow-up is required. The infection risks of transcutaneous wires are avoided.

Long-term pacing with an externally placed pulse generator enables the heart rate to be varied, the power source to be changed without further operations, and the power setting to be adjusted. The inductively coupled system (Abrams, Hudson, and Lightwood, I960; Abrams and Norman, I964; Taylor, I966) incorporates the advantages of an external pacemaker unit with endocardial pacing but avoids the high incidence of late electrode displacement and sepsis that occurs with the transcutaneous technique (Conway, Seymour, and Gibbons, 1969). We report here our first 33 months' experience with the Lucas system.

\section{Patients}

Forty-four patients (19 women and 25 men) aged between 44 and 82 years were paced with this system. Thirty-nine were paced with endocardial electrodes and 2 required ventricular myocardial wires. Three were given atrial pacemakers for sinus bradycardia, 2 of whom are described in detail elsewhere (Clarke, Evans, and Milstein, 7970). The remaining 4I patients had complete heart block which was intermittent in $1 \mathrm{I}$. Twentyeight patients were paced for Adams-Stokes' seizures, the other 16 because of symptoms due to 1 low cardiac output, 3 having had repeated ailure of totally implanted units inserted at other sentres.

Three patients had calcific aortic valve disease Ind I had calcific mitral stenosis. Two had been reated for myxoedema for some years, 2 had Iddison's disease, and 2 were diabetic. Three had hronic heart block associated with ischaemic eart disease. Another had Turner's syndrome. le remainder had no demonstrable pathology :eived Is June 1970 . apart from their heart block. Two patients have developed diabetes after over 2 years of pacing.

\section{Pacemaker equipment}

The standard Lucas inductively coupled cardiac pacemaker equipment has been used throughout. The only implanted component is a flat, ringshaped coil with the attached electrodes (Fig. I). The stimulating impulse is induced in this coil across the intact skin from an external coil taped to the patient's chest over the internal coil. The impulse energy can be reduced by offsetting the two coils and an additional switch inside the pulse generator (Fig. 2) can be used to double the impulse voltage if required.

The pulse generator (Fig. 3) is powered by the universally available ' $\mathrm{U}_{2}$ ' (or SP2) dry battery. The heart rate is adjusted by means of a rate control wheel on the front of the unit. An audible tick is produced with each impulse to enable the patient to check his heart rate with that of the pacemaker, but a silencing switch is provided. Any electrical fault in the plug, lead, or external coil causes a persistent buzzing tone to be emitted from the unit, thus effectively warning the patient of malfunction from this cause. The characteristics of the pacing stimulus are shown in Fig. 4. The energy of the stimulus remains constant throughout the life of the battery. As the battery runs down the pulse rate decreases and is adjusted by the rate control. The battery is replaced by substituting the spare generator (see below) when the required heart rate can only be maintained with the rate control at its maximum.

Each patient is given a spare pacemaker unit and external coil and a comprehensive booklet of instructions is provided.

\section{Operation}

Induction of anaesthesia is routinely preceded by transvenous placement of a temporary pacing 


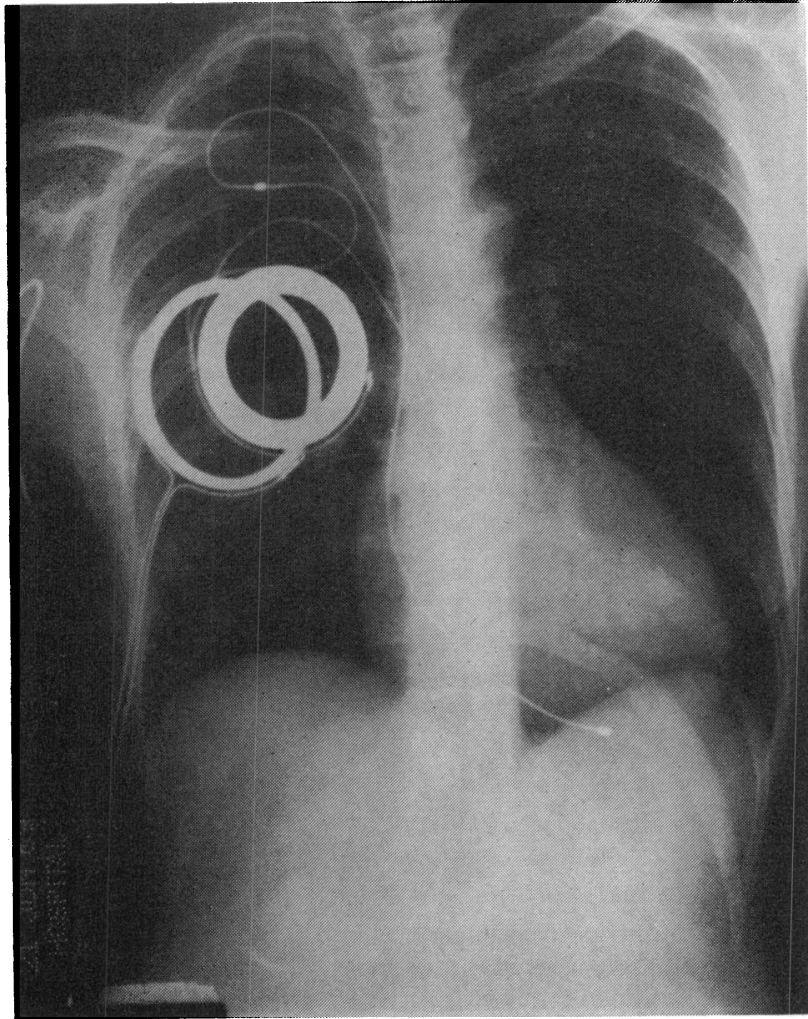

(a)

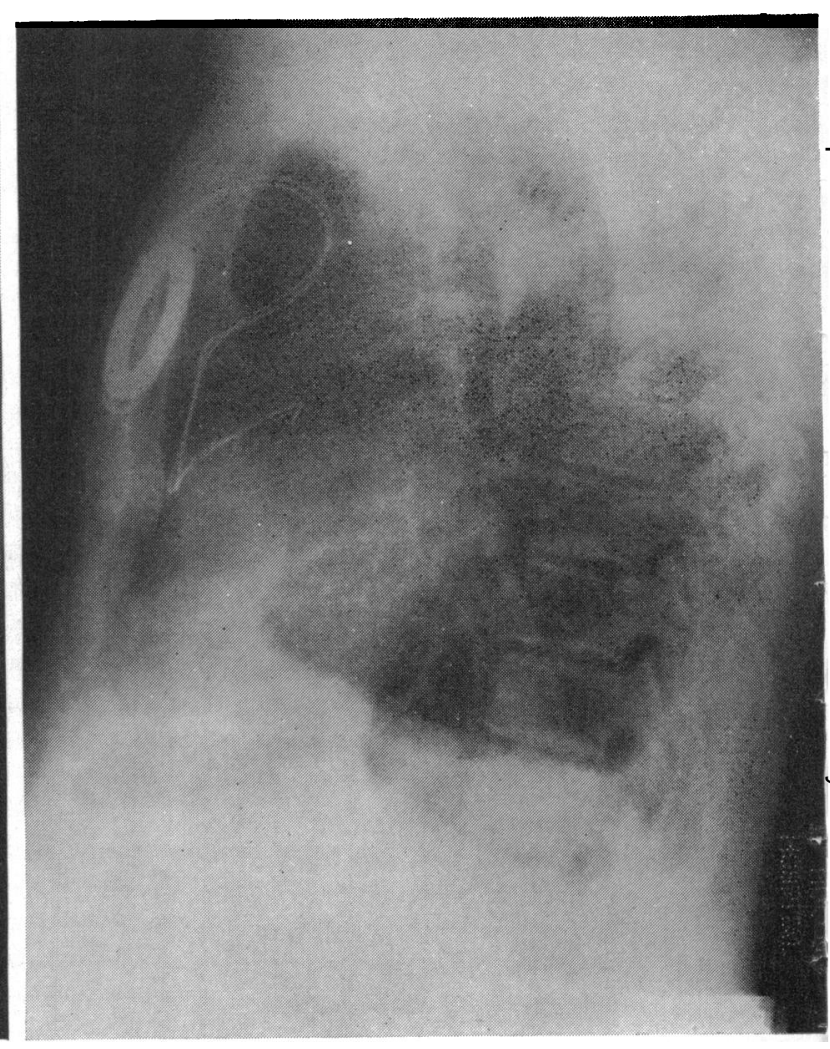

(b)

FIG. I Postero-anterior chest $x$-rays showing the implanted components of the inductive coupling system. (a) With endocardial electrode - the primary coil is in position on the skin over the (thicker) internal coil. (b) With ventricular myocardial electrodes.

electrode. This is connected via apposed primary and secondary coils to a standard 'Lucas' pacemaker, placed $183-244 \mathrm{~cm}$. from the patient. The electrocardiogram is monitored throughout and diathermy is routinely used.

With the patient placed left side down on the $x$-ray table (see below) a curved incision to to $15 \mathrm{~cm}$. long is made just below the clavicle extending downwards at its medial end parallel to the edge of the sternum for a short distance. A not too generous subcutaneous pocket for the secondary coil is dissected superficial to the pectoralis major muscle above the main bulk of the breast and medially rather than laterally, especially in those with rather lax tissues. The right internal jugular vein is then exposed for a short distance between the two heads of the sternomastoid muscle. Two purse-string sutures of $5 / 0$ mersilene are inserted in the wall of the vein enclosing an area $7 \mathrm{~mm}$. in diameter. A small puncture is made in the centre of this area for the insertion of the platinum-tipped electrode which is positioned in the apex of the right ventricle under screening control. A removable stilette is inserted into the electrode to facilitate this manoeuvre; with the patient in the position described, it has been found unnecessary to alter the natural curve of the stilette except occasionally to accentuate its bend (in the same plane) approximately $10 \mathrm{~cm}$. from its tip. If for any reason it is necessary to carry out this electrode placement with the patient supine, it is found advantageous to bend the last Io $\mathrm{cm}$. or so of the stilette through approximately $90^{\circ}$ counterclockwise looking caudally, and to accentuate the forward bend so as to direct the electrode through the tricuspid valve. However, if the $x$-ray facilities are adequate for screening in the lateral posture, it is a considerably easier and quicker task to achieve satisfactory electrode placement. The latter is signified by forward (and preferably slightly downward facing) electrode tip position, appearing in the lateral projection to be situated just behind the lower end of the sternum (Fig. 5). There should be a gentle continuous curve through the atrium. The postero-anterior view (obtained by radiography when screening is carried out in the lateral position) should show a few centimetres of the electrode to the left of the tricuspid valve position, again with only a gentle curve through the atrium and no gross buckling in the region of the tricuspid valve or elsewhere (Fig. ra). The position is not accepted unless the 


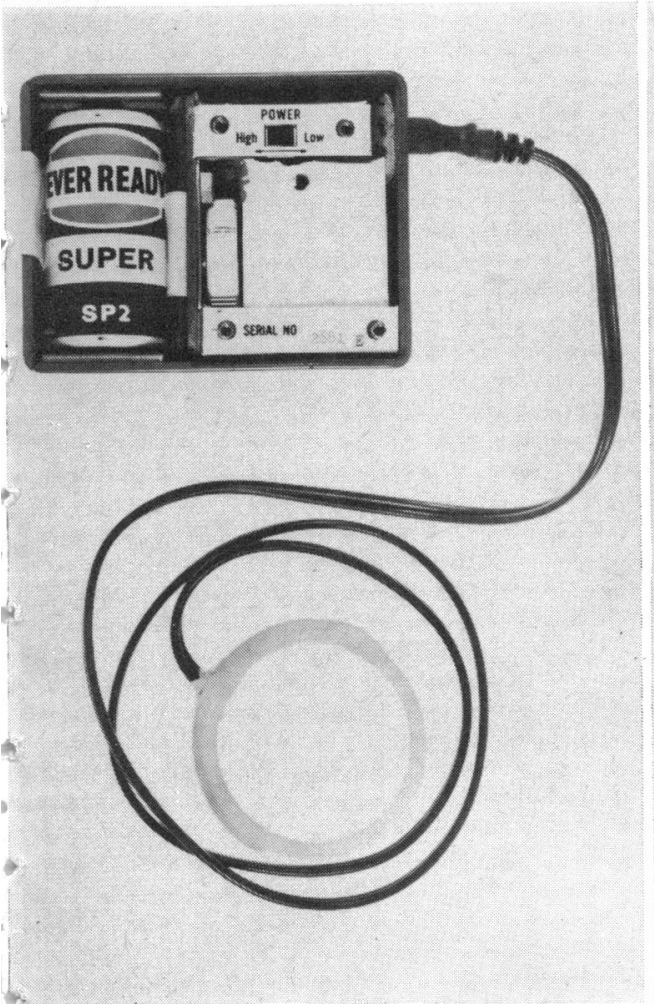

FIG. 2 Back of generator with the covers removed. The power setting is revealed by removing four screws and is not easily accessible to the patient.

$*$

pacing threshold as measured by a calibrated jacemaker is substantially less than I volt usually 0.3 to 0.7 volt) with a pulse width of imsec. Stability of the electrode tip is checked by icreening observation during simulated deep sreathing, produced by the anaesthetist, and by shecking that pacing is continuous throughout itise manoeuvres. Further checks can be made vith the stilette withdrawn to the atrial side of the "icuspid valve, and, if satisfactory, the stilette is hen withdrawn to the region of the superior vena ava and the indifferent loop electrode is sutured $\checkmark$ the periosteum overlying the sternum. The acing electrode is firmly fixed at the site of entry to the vein by tying both the purse-string tares and then tying each to the electrode. The scondary coil is connected to the electrode by teans of a push connector. Threshold is now zecked with a primary coil (in a sterile polythene dg) placed above the secondary, pacing at 4 to $\mathrm{cm}$. vertical displacement being required. With ontinuing induction-pacing as required, the mporary electrode is removed and, after further ureshold checks, the incision closed.

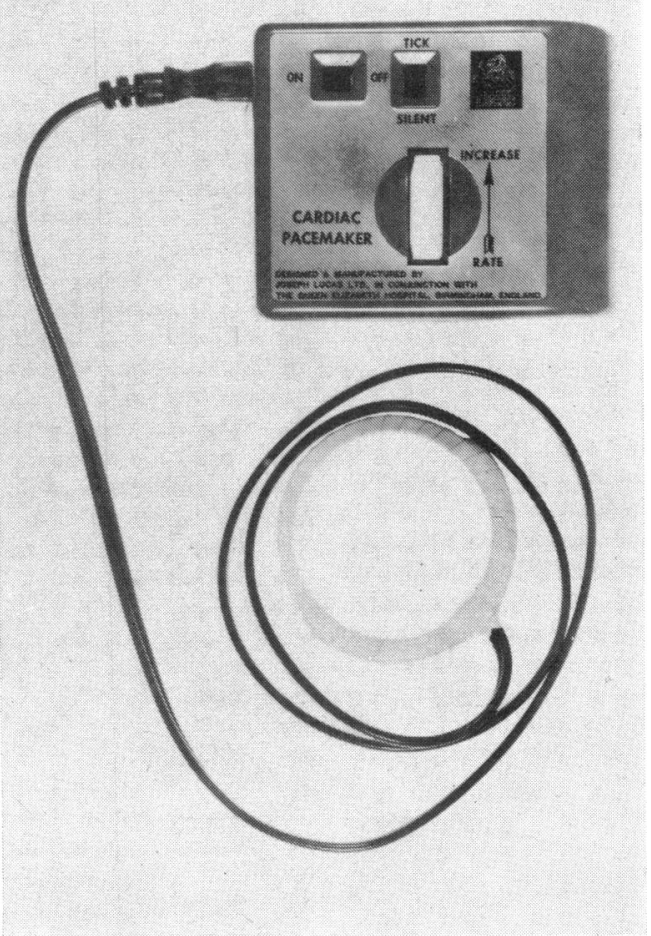

FIG. 3 Pulse generator and primary coil. The generator measures $10 \times 7 \times 4 \mathrm{~cm}$. The coil lead is $85 \mathrm{~cm}$. long.

FIG. 4 Stimulating pulse form. Coils at I $\mathrm{cm}$. separation, heart load I80 Ohms. The pulse voltage is doubled when high energy output is selected.

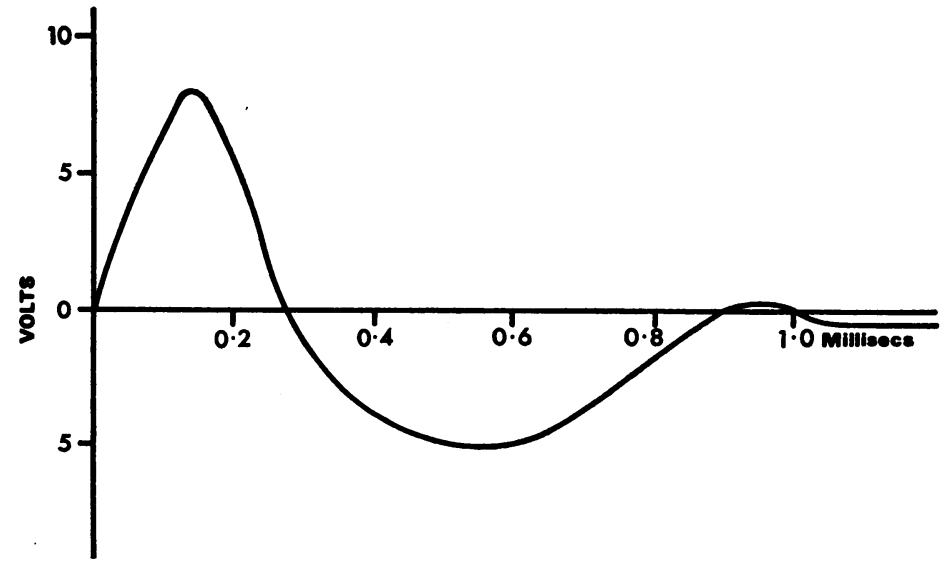


Formal pacing is initiated with the primary coil submaximally offset, i.e. up to half the secondary coil diameter, to minimize pacing energy delivered to the endocardium in the early postoperative period.

A lignocaine infusion is continued for 48 hours, or longer if there is ectopic activity. Oral procainamide may be given subsequently for two weeks or more. The patient is encouraged to lie on the left side and semipropped up for the first two postoperative days to assist electrode stability.

\section{Patient instruction}

It is usual to begin the patient's instruction in battery changing and coil positioning before his operation. Though it was initially felt that a high degret of intelligence would be required to cope with this system, there have been few real difficulties in practice; to some extent the increase in mental alertness that frequently occurs when pacing starts has been of help here. Three elderly women (two of them octogenarians) need help from relatives when changing the coil. As an added safety factor, relatives of all patients given this system are instructed in its use.

The pulse is checked twice daily and the heart rate adjusted as necessary. In practice, patients quickly learn to do this and they are proficient at battery and coil changing within a few days of receiving the system. To change the external components the patient sits in front of a mirror. The spare generator containing a fresh battery is connected to the spare lead and coil and set to the required rate. The old strapping is detached and the primary coil held in position with the fingers. With the other hand the coil of the new unit is then placed over the internal coil as the first primary coil is moved away. Continuity of pacing is thus maintained. The new coil is strapped to the skin in a slightly different position to avoid damage to the skin, optimal eccentricity or 'offsetting' being sought if muscle twitch is a problem and always providing that uninterrupted pacing under all normal conditions is assured.

This procedure is required approximately every four weeks when pacing on the low-power setting. In some patients with sensitive skin, more frequent coil repositioning may be desirable, and porous surgical tape may have to be used in place of the waterproof plastic adhesive strapping normally used.

\section{Patient follow-up}

Patients are visited at home by a technician within a month of discharge from hospital. They are reviewed as outpatients at 6 months and then at intervals of 12 months, when postero-anterior and lateral chest $x$-rays may be taken. The pacing threshold is simply measured by assessing the limits of coil displacement at which pacing continues. No electronic testing is necessary and no extra technical staff have been required to date.

A 24-hour telephone service is maintained for patients should they require help. Faulty coils and generators are replaced through the post.

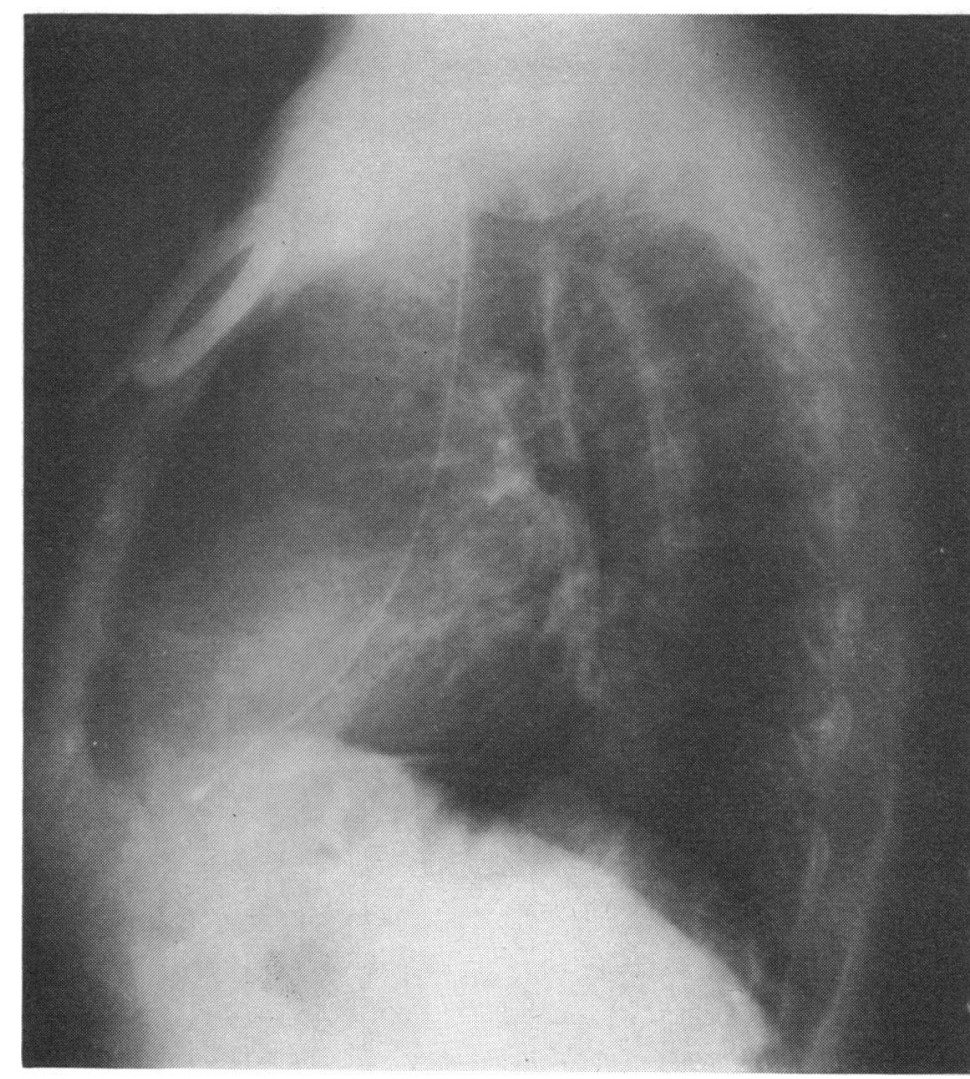

FIG. 5 Lateral chest $x$-ray to show position of endocardial electrode.

\section{Results}

Forty-four patients have been paced for periods of I to 33 months. Total experience covers 65 I patient-months.

Deaths Five patients have died. One, who had been paced for 9 months, had major pulmonary embolism after leg vein thrombosis. Another died suddenly while in gross cardiac failure after massive cardiac infarction which had necessitated the pacing 4 months earlier. A third died suddenly (at 73 years of age) at home after several days' malaise with headache and joint pains. The fourth death also occurred suddenly at home after malaise for one week. Pacemaker-induced ventricular fibrillation could not be excluded in the latter two cases, though competition is unlikely to have occurred in either, as the basic rhythm had been complete heart block with ventricular rates substantially below 40 a minute in both throughout several months of pacing and before operation.

One early postoperative death occurred. 
This was due to ventricular fibrillation in the early hours of the morning and attempts at resuscitation were delayed and unsuccessful.

" The arrest occurred on the fifth day, and after oscilloscopic monitoring and antidysrhythmic drug therapy had been withdrawn. Satisfactory pacing without competition or ectopic activity had been observed during the preceding 48 hours. As a result of this experience suppressive drug therapy is now continued

- for two or three weeks after operation.

External component deficiencies One generator has developed major technical - failure. In 3 others the battery clip became faulty. One patient's pulse generators had to be specially sound insulated because his - expensive pet dog was troubled by their very high-pitched emissions, almost inaudible to the human ear.

Twenty-three external coils have been replaced because of fracture of the leads, always at the site of origin of the leads from the plug. This point in the system has recently been - modified in an attempt to overcome this fault. When it has occurred some patients have suffered momentary faintness or 'dizziness' but none has suffered a major syncopal attack. -More frequently, no such inconvenience has attended development of this fault because it has been recognized (by production of the warning 'buzz' from the unit) immediately the plug has been fitted to the pulse generator and the latter switched on, i.e. before the newly-charged system has been substituted for the one actually in use.

Flectrode stability and secondary coil migration In 16 patients the endocardial - electrode was repositioned within the first 2 or 3 days. This high incidence of repositioning was in part due to lack of appreciation of the fact that there may be a sharp but temporary rise in apparent pacing threshold (as measured by the maximum vertical distance of the primary coil, from the skin over the secondary scoil, at which pacing continues) in the early postoperative period. This rise is probably due to effusion which collects about the secondary coil, and which may rarely require aspiration, and perhaps to subendocardial oedema in the region of the electrode tip. Now that this phenomenon is known, it is not considered necessary to undertake early repositioning on grounds of rising threshold alone, i.e. unless pacing failure threatens, because reversal of the threshold trend within a week may normally be anticipated.

Another factor contributing to the relatively large number of early repositionings is a degree of fastidiousness about proper placement. The electrode has to 'look right' on radiography or fluoroscopy, any significant displacement or continuing instability being unacceptable. It is thought that this policy may be the reason for comparative freedom from the problem of electrode displacement after discharge from hospital.

In one case a second repositioning of the electrode was necessary because of recurrent gross displacement while still an inpatient, but subsequent progress was free of this complication. In 2 instances it was necessary to abandon endocardial pacing and resort to myocardial wires. In the first patient this was due to right ventricular intolerance of the electrode which was rapidly returned to the right atrium from any location tried. In the second case there was very rapid appearance of 'exit block' progressing to intermittent pacing failure during the first week.

One patient had displacement of the electrode a month after leaving hospital. This was due to the electrode being inadequately secured at its entry into the internal jugular vein. The indifferent loop electrode uncoiled and projected through the skin in an elderly woman; it has now been satisfactorily replaced.

Repositioning of the secondary coil was required in one patient after it had migrated into the breast tissue a month after operation. In another, more medial repositioning of an unduly mobile secondary coil was necessitated by difficulty in maintaining full coupling during the involuntary movements of sleep.

In one very small patient - only $137 \mathrm{~cm}$. in height and $31.75 \mathrm{~kg}$. in weight - the secondary coil has extruded through the skin and is due to be replaced by the smaller paediatric coil.

Sepsis Only one serious wound infection occurred. This was due to a sensitive Staphylococcus aureus, and was cleared by systemic and local penicillin. This and 2 other wounds required resuture. All other wounds healed uneventfully.

No episode of septicaemia was encountered.

The operative period was covered routinely by a 7 to Io day course of either ampicillin orally or cephaloridine intramuscularly.

Long-term results All 39 survivors are active within the limitations of their years and are free from Adams-Stokes' attacks. They have required no special supervision apart from their outpatient review.

Three female patients have complained of difficulty in carrying the bulky generator but 
most have overcome this problem in various ingenious ways. No objections have been voiced in regard to the aesthetic disadvantages of strapping the primary coil to the skin, though some comment on the inconvenience involved when taking a bath.

Three patients who had previously had totally implanted pacemaker units volunteered preference for the inductive coupling system and particularly appreciate the safety factors of an external generator.

No patient has had any serious mishap while changing the external coil or generator, though one of the younger patients initially had trouble in accurately locating the primary over the secondary coil.

\section{Discussion}

Long-term pacing can be effected with internal or external generators using endccardial or myocardial electrodes. With an external generator the impulse can be transmitted to the heart by an electrode passing through the skin, or across the intact skin by radio-frequency transmission (Glenn et al., 1959), or by the simpler method of inductive coupling, developed in this country by the Birmingham group (Abrams et al., 1960).

Endocardial electrodes are now used as the method of choice by most workers. Thoracotomy with its attendant risks (Chardack et al., I966) is avoided and the high incidence of postoperative ventricular fibrillation reduced. However, one must still be prepared, as in 2 of our cases, to use the transthoracic method in certain instances (Morris et al., 1967; Goldstein et al., 1970). The inductive coupling system can be used with both methods, and its advantages lie in the use of an external generator.

Though many workers in this country prefer totally implanted units, the reported results are disappointing because of technical failures (Harris et al., I968; Goldstein et al., I970). In a large survey of implanted pacemakers published two years ago it was noted that generator failure accounted for 57 per cent of pacing failures. One-third failed before the generators were due to be changed (Sowton, 1968). Another cause of technical failure was fracture of the U.S.C.I. C.50 electrode, but most systems, including the inductive coupling method, now utilize a helical coil multistrand electrode with greatly improved reliability (Siddons and Sowton, 1967a). In the inductive coupling system, the only implanted parts are this electrode and a simple secondary coil, so there is little that can go wrong internally.
Implanted units are liable to fail or to discharge repetitively at very high rates without warning. A 'runaway pacemaker' presents as an emergency with ventricular tachycardia or fibrillation as a common occurrence. There is a high mortality in the most ideal conditions (Siddons and Sowton, 1967b). As our patients are dispersed over a wide area, and several are 70 or 80 miles away with poor road communications, such an emergency could be disastrous, and the use of an external generator is clearly preferable at this time.

In addition to protection from unpredicted battery failure, the external unit has the advantages of variable rate and power settings. As inductive coupling cannot at present be used with a 'demand' pacemaker, patients in sinus rhythm are overpaced at faster speeds in order to capture the rhythm and suppress sinus beats. This compromise works well in practice. Digoxin and propranolol are sometimes used in this situation, the former to increase the degree of atrioventricular block where this is unstable, and the latter to suppress the rate of sino-atrial node discharge where this is leading to competition. The availability of power change has been of value in 2 patients who have experienced a rise in pacing threshold and are now satisfactorily paced with the generator in the 'high power' mode. Such problems of developing 'exit block' are common to all pacing systems. In 3 patients an excessive rise in threshold has been observed during the first week of electrode placement, and this trend has seemed to reverse during shortterm steroid administration.

The Lucas generator is fitted with an $\mathrm{ON} / \mathrm{OFF}$ switch. The facility to discontinue pacing can occasionally be of value, as in one of our patients who developed transient atrial fibrillation after insertion of an atrial pacemaker (Clarke et al., 1970).

Though presently available implanted generators may have a useful life of approximately 2 years, a young patient may still require a series of Io or more operations in his lifetime to replace the units as they fail (Goldstein et al., 1970). Transcutaneous pacing offers the advantages of an external generator but has a high incidence of sepsis and late electrode displacement (Conway et al., 1969). With the inductive coupling system, late electrode displacement is rare; displacement of a firmly fixed electrode has only occurred within the first 48 hours, a similar experience to that found with other methods of pacing (Bluestone et al., I965).

A major disadvantage of implanted generators and transcutaneous pacing is the need for 
frequent, special assessment, which can be inconvenient to patients, and requires an increased establishment of skilled technical staff involving extra cost. These factors limit the successful use of these methods of pacing to one or two centres in the United Kingdom (Whitaker, 1970).

The disadvantages of the inductive coupling system include the requirement for taping the coil to the skin which is somewhat un-

- satisfactory and could prove a major disadvantage in the tropics. The patient has to take care to avoid getting the bulky generator wet while having a bath and, in general, the satisfactory use of the system depends on adequate patient co-operation. The possibility that electromagnetic fields may interfere with

- pacemaker function has been considered and local ophthalmology departments that use powerful electromagnets for removal of foreign bodies from the eyes have been

- warned of this potential risk. A more serious disadvantage in some cases is the lack of a ventricular-inhibited or other demand facility.

- This deficiency caused us to install a totally implanted demand system in a 63-year-old man with complete heart block and frequent ventricular ectopic beats, which were not

- abolished by pacing or reliably suppressed by antidysrhythmic drugs. Another patient has been given a totally implanted system because of his 89 years and the fact that he lives alone. A third patient was thought unlikely to be able to cope with coil changing, etc. because she was so severely crippled by rheumatoid disease. She was therefore given a totally implanted system 7 weeks ago, the generator tof which failed 7 days later: she was syncopal at this time but idioventricular rhythm emerged and she recovered. A new unit was implanted a few hours later and has func: tioned satisfactorily to date.

*We are most grateful to Mr. R. Lightwood of the Department of Surgery, Queen Elizabeth Hos- pital, Birmingham, for his invaluable help and practical advice in establishing the technique at this centre. The Lucas Research Laboratory has contributed much encouragement and maintained a prompt and helpful service.

We should like to thank Mr. John Prior, Mr. -Tim Cole, and the other cardiological technicians for their enthusiasm and hard work which makes this pacemaker service possible.
We are grateful to Mr. Christopher Parish, Consultant Thoracic Surgeon at Cambridge, and to the thoracic surgical registrars who performed some of the operations. We thank Dr. H. A. Fleming and the many other physicians who referred patients for pacing.

Fig. 4 is reproduced by permission of the Lucas Medical Research Department.

\section{References}

Abrams, L. D., Hudson, W. A., and Lightwood, R. (1960). A surgical approach to the management of heart-block using an inductive coupled artificial cardiac pacemaker. Lancet, $1,1372$.

$\longrightarrow$, and Norman, J. C. (1964). Experience with inductive coupled cardiac pacemakers. Annals of the New York Academy of Sciences, III, 1030.

Bluestone, R., Harris, A., Davies, G., Leatham, A., and Siddons, H. (I965). Results of long-term endocardial pacemaking with implanted units. In Proceedings of the British Cardiac Society. British Heart fournal, 27, 946.

Chardack, W. M., Gage, A. A., Federico, A. J., Schimert, G., and Greatbatch, W. (1966). The long-term treatment of heart block. Progress in Cardiovascular Disease, 9, 105.

Clarke, M., Evans, D. W., and Milstein, B. B. (1970). Sinus bradycardia treated by long-term atrial pacing. British Heart fournal, 32, 458.

Conway, N., Seymour, J., and Gibbons, D. (1969). Long-term transcutaneous endocardial pacing in management of complete heart block. British Heart fournal, 31, 163.

Glenn, W. W. L., Mauro, A., Longo, E., Lavietes, P. H., and Mackay, F. J. (1959). Remote stimulation of the heart by radiofrequency transmission. Clinical application to a patient with Stokes-Adams syndrome. New England fournal of Medicine, 26r, 948.

Goldstein, S., Moss, A. J., Rivers, R. J., Jr., and Weiner, R. S. (1970). Transthoracic and transvenous pacemakers. A comparative clinical experience with I3I implantable units. British Heart fournal, 32, 35.

Harris, A., Redwood, D., Davies, M., and Davies, G. (1968). Cause of death in patients with complete heart block and artificial pacemakers. British Heart fournal, 30, 14 .

Morris, J. J., Whalen, R. E., McIntosh, H. D., Thompson, H. K., Brown, I. W., and Young, W. G. (1967). Permanent ventricular pacemakers comparison of transthoracic and transvenous implantation. Circulation, 36, 587.

Siddons, H., and Sowton, E. (I967a). Cardiac Pacemakers, p. I19. Charles C. Thomas, Springfield, Illinois.

- and - (1967b). Cardiac Pacemakers, p. ro3. Charles C. Thomas, Springfield, Illinois.

Sowton, E. (1968). Result of a British survey on artificial cardiac pacemakers. British Medical fournal, 2, II.

Taylor, A. B. (1966). Experience with cardiac pacemaking. British Medical fournal, 2, 543.

Whitaker, W. (1970). Cardiology. World Medicine Review of the Year, February 1970, p. 5I. 